\title{
Carnets
}

Revue électronique d'études françaises de l'APEF

Première Série - 2 Numéro Spécial | 2010

Littératures nationales: suite ou fin. Résistances, mutations \& lignes de fuite

\section{Litterature migrante comme lieu de construction de cultures de convergence}

\section{Gilberte Février}

\section{(2) OpenEdition}

\section{Journals}

Édition électronique

URL : http://journals.openedition.org/carnets/4860

DOI : $10.4000 /$ carnets.4860

ISSN : 1646-7698

Éditeur

APEF

Édition imprimée

Date de publication : 1 juin 2010

Pagination : 27- 41

Référence électronique

Gilberte Février, «Litterature migrante comme lieu de construction de cultures de convergence », Carnets [En ligne], Première Série - 2 Numéro Spécial | 2010, mis en ligne le 16 juin 2018, consulté le 21 décembre 2020. URL : http://journals.openedition.org/carnets/4860 ; DOI : https://doi.org/10.4000/ carnets. 4860

Carnets est mis à disposition selon les termes de la licence Creative Commons - Atribution - Pas d'utilisation commerciale 4.0 International. 


\title{
LITTERATURE MIGRANTE COMME LIEU DE CONSTRUCTION DE CULTURES DE CONVERGENCE
}

\author{
GILBERTE FEVRIER \\ Université du Québec à Montréal \\ gilbertefevrier@hotmail.com
}

\begin{abstract}
Résumé
Dans cette recherche, nous avons voulu montrer que la littérature migrante est construction de cultures de convergences. En nous appuyant sur le roman Passages (1992) d'Émile Ollivier, nous avons analysé les caractéristiques de la littérature migrante et essayé de montrer son inscription dans le courant postmoderne. Nous avons donc traité d'identités multiples, d'appartenances culturelles et de langues métissées.
\end{abstract}

\section{Abstract}

This research aims to show that migrant literature is reflecting and building convergent cultures. Using Passages (1992), a novel by Émile Ollivier, we analysed the characteristics of migrant literature and tried to show its appurtenance to the postmodernist stream. We therefore discussed multiple identities, cultural backgrounds and mixed languages.

Mots-clés: Littérature migrante, Identité, Appartenances multiples, Exil, Ancrages et métissages linguistiques, Postmodernisme

Keywords: Migrant literature, Identity, Multiple affiliations, Exile, Anchors and language mixing, Postmodernism 
Quelles que soient les qualifications qu'on lui attribue, les circonstances qui le vouent à bouger ou la distance du trajet parcouru, l'être humain depuis la nuit des temps, se déplace d'un coin à l'autre de la planète. Aussi, les quarante dernières années du vingtième siècle ont-elles été témoins d'une vague d'immigration de professionnels venus de l'Amérique latine et des Caraïbes pour des raisons politiques, sociales et économiques. Les sciences humaines ont fait de la diaspora un champ de recherche à part entière. Selon les chercheurs, elle constitue l'un des indicateurs sociaux qui permet d'envisager et d'expliquer les différents rapports que les humains entretiennent entre eux et avec la société, sans parler de leurs relations avec l'État (Bruneau, Berthomière et Chivallon, 2006), notamment en ce qui concerne l'allégeance, le retour éventuel, le nationalisme, l'appartenance, l'exil et l'identification pour ne citer que ces aspects. L'évolution du discours identitaire résultant de ces changements géographiques et démographiques génère des changements littéraires et langagiers au niveau des communautés diasporiques. Les écrits issus de cette mouvance, qualifiés d'écritures migrantes (Berrouet-Oriol, 1986; Moisan et Hildebrand, 2001 et Prud'homme, 2002), selon certains critiques, se distinguent par une série de spécificités thématiques, génériques et langagières particulières, et se définissent dans une dynamique littéraire plurielle, caractérisée par la multiplicité, l'ambiguïté et l'interstice (Harel, 2005; Robin, 1992). Autrement dit, ces écrits sont une construction de cultures de convergence.

Dans le présent article, nous nous proposons d'analyser la littérature migrante comme élément clé de l'anamnèse, pour ensuite dégager ce qui peut bien caractériser ce sous-champ littéraire, c'est-à-dire, l'inscription de ces écrits dans la postmodernité; leur tissage avec d'autres textes (intertextualité) et la pluralité de langues dont ils sont faits. Notre travail se basera particulièrement sur les écrivains migrants Haïtiens au Québec, spécifiquement Émile Ollivier (1940-2002). Toutefois, de temps en temps, nous ferons appel à d'autres écrivains migrants pour illustrer notre propos.

\section{Horizons multiples, nouvelles formes d'expression}

\subsection{Vague d'immigration}

L'exil et la mondialisation ont déclenché une vague d'immigration politique, sociale et économique, notamment aux États-Unis et au Québec. Cet éloignement géographique qu'ont connu de nombreux professionnels et intellectuels (politiciens, médecins, ingénieurs, enseignants, intellectuels, etc.) n'a pas rompu le lien qui les unissait à leur pays: chacun, à sa manière, a emporté et apporté sa patrie avec lui. Jetons un regard sur quelques écrivains migrants du Québec. 


\section{2. Écrivains migrants du Québec}

Naïm Kattan: Bagdadien qui émigre à Montréal en 1954. Dans ses œuvres, il se propose d'aller à la rencontre de cultures différentes, de réfléchir sur les rapports humains et leur diversité. II est, selon les critiques, un carrefour des cultures à lui seul. La fiancée promise, publié en 1983, décrit Montréal, cette ville dont les villages sont adossés les uns aux autres, et où se croise une pluralité de communautés linguistiques et religieuses. Aux yeux de l'enfant de Bagdad, c'est une ville du Moyen-Orient.

Dany Laferrière: Haïtien, entré au Québec en 1976. II quitte Montréal pour vivre en Floride pendant dix ans. Depuis 2000, il s'est réinstallé à Montréal. Dans ses œuvres, il revient sur les traces de son passé, de ses origines. Dans L'énigme du retour. (2009), Prix Médicis, il aboutit à une réflexion sur l'exil.

Ying Chen: née à Shanghai, elle s'installe à Montréal en 1989. Elle publie de nombreux romans dont Les lettres chinoises (1993). Dans cette œuvre, une correspondance s'établit entre un jeune immigrant et sa fiancée restée en Chine. Le roman témoigne du choc des cultures, du déracinement et de l'impossibilité de l'amour.

Émile Ollivier: né en Haïti, est entré au Québec en 1965. Dans Les Urnes scellées (1995, Prix Carbet de la Caraïbe), il traite de la possibilité du retour. Dans ce récit, Adrien Gorfoux, archéologue, et sa femme Estelle, exilés au Québec, retournent en Haïti après la fuite du couple Duvalier en 1986. C'est le fruit d'une réflexion sur l'exil, ou plutôt l'errance, les choix à faire, et l'impossibilité de résoudre l'Histoire d'Haïti.

Marco Micone: né à Montelongo en Italie, arrive à Montréal en 1958, à l'âge de 13 ans. La plupart de ses œuvres (théâtre, romans, poésie) traitent de la condition immigrante et luttent contre l'intolérance ethnique et linguistique. Micone s'intéresse au dialogue interculturel entre les Italo-Canadiens et le reste de la population québécoise. Le figuier enchanté (1992), lui a valu le Prix des Arcades de Bologne en 1992. Le roman relate le processus d'acculturation et de construction de l'identité migrante.

Les œuvres de ces écrivains venus d'ailleurs se présentent sous une forme hybride, voire baroque (Ouellet, 2003). Les critiques littéraires les regroupent sous la dénomination de littérature migrante (Lebrun et Collès, 2007).

\section{Littérature de mouvements}

Simon (1994) nous rappelle les différentes dénominations que peut connaitre ce mouvement littéraire, selon l'espace géographique: Ethnic or Multi Ethnic literature, de littérature de l'exil ou de littérature des frontières. Qu'importent les différentes qualifications, 
toutes justifiables et justifiées, qu'on attribue à ce mouvement littéraire, il faut préciser qu'on le reconnaît grâce à la convergence des thématiques génériques et langagières spécifiques. Par convergence, nous entendons le fait de tendre vers un seul et même point, un seul et même but. Ces thématiques témoignent toutes de mouvements, de brassages, de convergences, de rencontres et de quêtes identitaires multiples (Ollivier, 1984). L'écrivain migrant, tout comme les personnages qu'il invente, se construit grâce aux différents mouvements et déplacements qu'il effectue; ces derniers peuvent être de nature géopolitique ou ontologique.

\subsection{Mouvement géopolitique}

Le migrant sort d'un lieu géographique (pays d'origine) pour se rendre vers un ou plusieurs lieux (pays d'accueil). Dans le cas d'Émile Ollivier, l'écrivain dont nous avons choisi l'œuvre, Passages, il a laissé Haïti, d'abord, pour la France; ensuite, il a immigré au Québec. Normand Malavy, un des personnages principaux de Passages, quittait de temps en temps Montréal pour ailleurs. II en va de même de Dany Laferrière, qui pendant longtemps, faisait le va-et-vient entre le Québec et la Floride avant d'y retourner définitivement en 2000. Ying Chen aussi a habité dans plusieurs grandes villes du Canada (Montréal, Vancouver, etc.). Tout se passe comme si le migrant ne savait pas où se fixer ou ne pouvait pas se fixer.

\subsection{Mouvement ontologique et symbolique}

Le migrant se questionne sur lui-même, sur ses valeurs, sur sa culture et sur son errance. Pour Émile Ollivier, ces mouvements constituent une sorte de migrance. Par là, il désignait le caractère inachevé, tant du côté psychologique que social de la migration. Quant à Lebrun (2001), elle parle de chantier, ce qui renvoie à la construction de soi et de son identité. Cet état témoigne d'un travail en perpétuelle construction, alors que l'immigration, concept couramment utilisé, présente un aspect réducteur, figé et dénote l'achèvement (Ceccon, 2004). Le migrant pense venir et n'être de nulle part partout, alors qu'il manifeste la volonté de s'intégrer (Camilleri, 2004). C'est au confluent de ces contradictions et de ces tensions qu'il tente de se construire (Forget, 2003). Cela dit, comme nous allons le montrer bientôt, les thèmes traités dans la littérature de la migration (exil, l'identité, double appartenance, etc.) reflètent cette confluence.

\section{Thématiques particulières}


Selon les chercheurs, la littérature migrante se construit principalement autour de quatre thématiques centrales: l'identité, la double appartenance culturelle, l'exil et la langue.

\subsection{Identité}

Terme polysémique, l'identité évoque la similitude, l'unicité, la permanence, la reconnaissance et l'individualisation (Rey-Debove, J., Cottez, H et Rey, L. 2005). Toutefois, le concept ne se résume pas aux aspects juridiques et linguistiques mentionnés ci-dessus. En sciences humaines (Lévi-Strauss, 1987), il caractérise l'homme dans sa complexité psychologique et sociale, dans ses relations avec l'autre, en permettant de l'envisager comme un élément d'un groupe social. C'est dans ce contexte que se justifie l'assertion qui fait de l'identité une définition de soi-même et d'autrui (Béra et Lamy, 2003).

Définir le concept revient à le placer au carrefour de plusieurs champs disciplinaires, notamment le droit, la psychologie, la philosophie, l'anthropologie et aussi la sociologie. Partant de ces champs de spécialisation, nous dégageons une identité personnelle et une identité sociale qui englobe l'identité culturelle. Cette dernière renvoie aux marqueurs identitaires, ces éléments prédéfinis qui permettent de distinguer les différentes cultures (Gerraoui et Troadec, 2000). II est important, à ce niveau, de souligner que les contacts qui s'établissent entre les cultures (le métissage culturel ou d'identité interculturelle) sont aussi l'occasion des acquisitions de connaissances d'une culture (inculturation ou acculturation).

En bref, l'identité est mouvante, en perpétuel changement et en construction perpétuelle. La culture d'un individu, tout comme son identité, n'est pas univoque. Elle est toujours convergence de plusieurs cultures et appartient au domaine de l'être et de l'avoir. Émile Ollivier, individu de sexe masculin, immigrant, Québécois, appartenait à la culture haïtienne. De plus, il était sociologue, professeur d'université et écrivain. Qui plus est, père de famille, grand lecteur et malade en hémodialyse, nous concluons qu'il avait une identité propre ce qui est le cas de chacun d'entre nous.

Cette identité, l'écrivain migrant tente perpétuellement de la reconstruire, à partir de difficiles négociations, dans un espace et dans des lieux où se croisent des appartenances multiples (Robin, 1992). Cela dit, l'identité migrante apparait sans cesse en évolution, en construction. Cependant, avant de présenter l'appartenance culturelle, arrêtons-nous sur la notion de culture.

\subsubsection{Culture}

L'éclairage que nous recherchons pour définir le concept de "culture" se situe au confluent de plusieurs traditions de pensée: littéraire, politique, philosophique, sociologique 
et, en particulier, anthropologique. Les anthropologues nous apprennent qu'elle fait partie du domaine de l'acquis et est "traces du milieu artificiel que l'homme a lui-même créé" (Moles, 1967: 19). Aussi, la culture englobe-t-elle l'ensemble des manifestations intellectuelles, religieuses et artistiques (les mœurs, les croyances, les coutumes, le droit, etc.) que l'homme s'est données en société. Par là, dégageons au moins, trois dimensions de la culture: une matérielle, une immatérielle et une corporelle (Galaty, 1991). C'est ce qui permet à un individu d'exercer dans la société les fonctions instrumentale, dialectique, ontologique et sociale (Billaud, 1996; Abdallah-Pretceille, 1999). Aussi, lorsqu'on parle de culture, ici, nous pouvons aborder le concept selon la signification que lui attribue l'Unesco, à savoir: “(...) l'ensemble des traits distinctifs, spirituels et matériels, intellectuels et affectifs, qui caractérisent une société ou un groupe social. Elle englobe, outre les arts et les lettres, les modes de vie, les droits fondamentaux de l'être humain, les systèmes de valeurs, les traditions et les croyances"' (Unesco, 1982: 10). Cela dit, qui sont les écrivains migrants? À quelles communautés appartiennent-ils?

\subsection{Appartenances culturelles multiples}

Le terme d'appartenance désigne le fait, pour un individu, de faire partie d'un groupe et surtout de s'identifier à celui-ci (Mucchielli, 1980: 99). Cela implique une identification personnelle, des attaches affectives, l'adoption des valeurs et des normes de ce groupe. L'appartenance permet à l'individu de s'intégrer, de s'actualiser en lien avec les autres membres du groupe (Campeau et al. 2004), d'exister, de construire et de se construire, de créer, de mettre en œuvre des projets capables de servir la collectivité (Boucher et Morose, 1990: 417). Bref, de pratiquer et de partager des valeurs civiques grâce à une certaine "cohésion" (Weinberg, Gould, 1997: 186). De ce fait, nous pouvons déduire que le "sentiment d'appartenance" est un processus interactif. Rappelons que ce sentiment se construit et se consolide peu à peu. Inversement, on peut faire partie d'un groupe sans pour autant s'y identifier.

Double ou multiple, cette appartenance, dans la littérature migrante, signifie cette rencontre de voix, auteurs, narrateur et/ou personnages venus d'horizons multiples, porteurs de cultures, de valeurs et de langues diverses (Ollivier, 1999). Pour Jean-Claude Charles (2001), l'écrivain migrant, ainsi que les personnages qu'il invente, concilient deux aspects contradictoires de leur situation, l'enracinement et l'errance: d'une part, il éprouve le besoin ou se fait un devoir de garder vivaces ses racines et, d'autre part, de rassembler les

Déclaration de Mexico sur les politiques culturelles. Conférence mondiale sur les politiques culturelles, Mexico City, 26 juillet au 6 août 1982. 
conditions d'une intégration réelle dans sa nouvelle patrie. Cet état d'être produit ce que Charles (2001: 37) appelle une enracinerrance.

En fait, le personnage ou l'auteur migrant se définit d'abord autour d'une conscience, en fonction de représentations de soi et à partir de pressions culturelles qui viennent à la fois du milieu dans lequel il est né et de celui dans lequel il vit. Puis, par les valeurs, les croyances, les connaissances et les expériences particulières qu'il a vécues et vit dans son pays d'origine et dans son pays d'accueil. Ces dernières font partie intégrante de son identité; elles lui donnent un sens et il les revendique pour lui-même. C'est avec l'ensemble de ces aspects qu'il participe à la nouvelle collectivité qui est désormais la sienne. Cette convergence de cultures donne naissance, chez lui, à une identité interculturelle, c'est-à-dire qu'il s'approprie la langue et la culture de sa nouvelle patrie.

\subsection{Exil}

Autre thème fondateur de la littérature migrante, l'exil peut être forcé ou volontaire; vécu ou imaginé (Nepveu, 1999). Par la distance qui s'établit entre sa culture d'origine et lui, l'exilé, qu'il soit auteur ou personnage, peut prendre du recul. Aussi, en devient-il à la fois "dépositaire" et "témoin" (Kristeva, 1988: 25). Cette double posture lui permet d'apprécier sa culture: il peut la critiquer, l'idéaliser ou la relativiser (Kristeva, 1988). Il le fait par rapport à l'autre culture, elle-même à la croisée d'autres cultures. Chez les écrivains haïtiens exilés, cet héritage spirituel se traduit sous la forme d'un "réalisme merveilleux", c'est-à-dire une description exagérée ou dépassée de la réalité nationale.

Par là, la littérature migrante se veut un carrefour de cultures, un lieu de convergences, celui du pays natal de l'écrivain, particulièrement de son enfance et celles des cultures rencontrées en terre d'adoption L'expérience "exilaire", qu'elle soit "interne" ou "externe" (Fonkoua, 1993), est "une métaphore qui actualise le rapport précis de l'écrivain au temps et à l'espace", dont le point de ralliement est le "mouvement" (Ouellet, 2003: 10). Pour Senghor, l'exil produit une "bipolarité de la culture", c'est-à-dire un profond "enracinement" à la terre natale grâce à "l'héritage spirituel", mais aussi un "déracinement" qui génère des "ouvertures aux rapports fécondants des civilisations étrangères" (Senghor, 1993: 295).

\subsection{Ancrage et métissage linguistiques}

Le départ du pays natal provoque luttes, deuils et déchirements. En effet, le sentiment d'appartenance s'exprime par une difficile reconnaissance de nouveaux ancrages géographiques, identitaires et linguistiques. Comment le migrant fait-il face à "la langue de l'hôte", pour répéter Ollivier, ou pour dire comme Barthes, à "l'autre langue"? II est certain 
que la langue appartient aussi à des lieux politiques et idéologiques habitables ou imaginaires. Ces dernières mettent en scène une polysémie sociale et culturelle qui prend la forme d'un brassage contemporain de signes, d'identités, de mœurs, de coutumes et de langues. Ces espaces géopolitiques sont ceux du "bord", de l'"écharpe", du "boitement". Tel un "cavalier", l'écrivain migrant "traverse", "chevauche" et "offense" (Barthes, 1993: 200).

Les écrivains des communautés diasporiques du Nouveau Monde des États-Unis et du Québec sont souvent confrontés au problème de la langue. Ils n'ont pas nécessairement l'anglais ou le français comme langue maternelle. Ils sont obligés d'apprendre, selon le pays, la langue. En effet, l'écriture requiert de l'écrivain la capacité d'utiliser non seulement une langue orale, mais la capacité de le faire à l'écrit avec une certaine assurance dans sa culture d'adoption et de créer une ambiance qui prouve sa connaissance de la société en question.

Kattan, Chen, Laferrière et Ollivier n'ont pas échappé à cette pression ou contrainte, même si pour certains d'entre eux, le français n'est pas une langue étrangère, vu qu'ils ont connu leur scolarisation dans la langue de Voltaire. Ils n'entretiennent pas tous le même rapport au français. Si Émile Ollivier fait montre d'un français "austère", par contre Dany Laferrière le manie avec "désinvolture" et Naïm Kattan la fait chatoyer (Lebrun, 2001: 31). Face à cette assertion de Lebrun, nous nous demandons si la langue utilisée par le romancier ne varie pas en fonction de sa culture et du lectorat ciblé.

L'écrivain migrant est au moins bilingue; cependant il joue sur les registres de chacune de ces langues. C'est pourquoi si la langue est un outil de communication et constitue une composante identitaire, dans la littérature migrante, elle devient une thématique majeure.

\subsection{Langue à "reterritorialiser"}

Même si leur langue maternelle est le créole, Agnant, Laferrière et Ollivier, pour ne citer que ces romanciers haïtiens, ont un rapport particulier à la langue et à la culture françaises. Un retour sur l'histoire d'Haïti nous permettra de mieux les comprendre.

La République d'Haïti possède une superficie de $27.750 \mathrm{Km}^{2}$ (par rapport au Québec $7.487169 \mathrm{Km}^{2}$ ). Elle partage avec la République Dominicaine (dans la proportion d'un tiers, deux tiers) le territoire de l'île baptisée Hispaniola par les Conquistadores. La première, Haïti, située à l'ouest, est francophone; la seconde, la République Dominicaine, ancienne colonie espagnole, est hispanophone.

Peuplées à l'origine d'Amérindiens (Indiens venus d'Amérique du Sud) rapidement décimés par les armes et les microbes des Européens, les Antilles ont été colonisées au sens premier du terme. C'est-à-dire que l'Espagne, la France, l'Angleterre y ont établi des 
soldats, des missionnaires, des administrateurs et des indésirables. Par la suite, ces colons ont importé, contre leur gré, des Africains pour les faire travailler comme esclaves dans l'agriculture (Prat, 1986). Haïti, appelée alors Saint-Domingue, a été une colonie française très prospère tout au long $\mathrm{du} \mathrm{XVII}{ }^{\mathrm{e}}$. Puis l'écho des évènements révolutionnaires qui bouleversaient la France y a suscité une révolte d'esclaves sans précédent. Sous la direction de Toussaint Louverture, celle-ci devait déboucher en 1801 sur un statut de colonie autonome, que les Français, en 1802, ont tenté d'abolir. L'échec militaire qui s'en est suivi, pour eux, a ouvert la voie à l'Indépendance, proclamée le $1^{\mathrm{e}}$ janvier 1804.

Inévitablement, à cause de sa situation d'ancienne colonie française, Haïti est l'un des rares pays des Caraïbes dont une des deux langues officielles est le français. Ses voisins immédiats parlent espagnol ou anglais.

Pour les écrivains migrants haïtiens, leur langue est celle du maitre français. Ils continuent de se l'approprier, de la tropicaliser ou de la créoliser. En ce sens, ils réalisent un vœu ancien exprimé par des théoriciens haïtiens de l'École de 1836 dans leur manifeste et que Jacques Roumain et Jacques Stephen Alexis, deux auteurs majeurs de la littérature haïtienne, ont réalisé: brunir la langue sous le soleil des tropiques.

Étant donné qu'une langue porte et transporte en elle-même des valeurs grâce auxquelles nous percevons le monde et la place que nous y occupons, la langue française s'est trouvée enrichie par les multiples voyages que nos écrivains ont effectués de part et d'autre de l'océan. À ce propos, Émile Ollivier, insatisfait, refusait ce français tel qu'il l'avait connu en Haïti et trouvé au Québec. En effet, ce français était pour lui 'figé, aseptisé, sans cavalcade de cheval, sans crissement de charrette, sans bouse de vache (...) (Ollivier, 2002). Aussi a-t-il tenté, dans son œuvre, de "le gonfler de toutes les ressources oniriques, baroques". Il l'a fait "sortir", dit-il "de sa pauvreté" (Ollivier, 2001: 55).

Mentionnons que les écrivains haïtiens Agnant, Lahens, Trouillot, Laferrière et Ollivier ne sont pas les seuls à avoir fait face à cette problématique. Se sont également attaqués à ce défi des écrivains antillais comme Glissant, Chamoiseau, Carpentier, etc.

En conclusion, pour les écrivains migrants, la langue n'est donc pas seulement un outil de communication qui aide à échanger et à mettre en commun leurs idées, leurs pensées ou un objet qui les caractérise et qui leur permet de s'identifier à un groupe social, mais elle devient une thématique spécifique (Moisan et Hildebrand, 2001) comme nous l'avons déjà dit plus haut.

Nous avons vu que la littérature migrante se construit principalement autour de quatre thèmes centraux: l'identité, la double appartenance culturelle, l'exil et la langue. Ces thématiques ne sont pas univoques: elles portent et construisent des cultures. C'est dans cette optique que la littérature migrante est considérée comme "construction de cultures de 
convergence". De plus, ces caractéristiques font en sorte que cette littérature s'inscrit tout à fait dans la postmodernité.

\section{Postmodernisme comme construction de culture de convergence}

Le postmodernisme est un courant artistique qui a pris naissance vers la fin du XXème siècle, particulièrement dans les années 1980 (Hutcheon, 1988). II est une "déconstruction" des tendances de la modernité et la reconstruction d'une autre rationalité. Passages (1992) d'Émile Ollivier, peut être considéré comme un roman postmoderne. En effet, pour Boisvert (1995) l'œuvre postmoderne se reconnaît à partir d'un ensemble de caractéristique dont notamment une coexistence de différences, une construction de cultures; une pensée de l'errance.

Une caractéristique du postmodernisme est la "coexistence pacifique" des différences. Ces dernières se complètent sans pour autant perdre leur originalité. (Raulet,1989: 11) n'y voit pas un "consensus rationnel", mais plutôt "un compromis constant" qui se fait grâce "aux jeux des langages" (idem, 21). Cette coexistence n'est exempte ni de conflits ni de souffrances. Ils sont là, certes, mais ceux-ci sont gérés et deviennent sources de socialisation.

En effet, toute coexistence de cultures est inséparable de situations conflictuelles. Autant elle soulève des tensions et des déchirements, autant elle pacifie les "conduites individuelles ou collectives”. (Lipovetsky, 1987: 229) et permet une adaptation. Grâce à cette situation des cultures lointaines les unes des autres se rapprochent en faisant tomber certaines barrières et hiérarchies. La coexistence des contraires apporte la force, la démocratie, la diversité. A titre d'exemple, citons la société québécoise que nous connaissons davantage après notre pays d'origine. Le postmodernisme favorise la pluralité, la cohabitation des appartenances multiples. En quoi Passages est-il représentatif de ce courant?

\subsection{Passages et le postmodernisme: coexistence de différences}

Des citoyennetés multiples (Québécois, Américains, Cubains, Jamaïcains, Haïtiens, etc.) se croisent et se cherchent dans ce pays qui les a accueillies. Déracinés, perdus dans des univers qui ne leur appartiennent plus ou pas, les personnages de Passages sont tous traversés par cette impression d'être de passage. Ainsi, ils nourrissent le rêve du retour au pays d'origine. Le Québec ne suffit pas à apporter à Normand toute la quiétude d'esprit dont il a besoin pour vivre. En dépit des difficultés rencontrées au début comme tout migrant dans une nouvelle ville, il s'y installe avec sa femme. 
Toutefois, il est hanté par la perspective du retour. II souhaite revoir Haïti et tout ce qui la pare, la peuple et la rend unique et paradisiaque. Ses villes lui manquent, particulièrement Port-au-Prince, "ville souveraine", "ville carrefour", "ville bigarrée", "terre auguste et roturière" (Passages: 75). Faute de pouvoir s'y rendre, il erre, effectue de nombreux va-et-vient entre le Québec et la Floride pour se ressourcer. Amparo, sa maîtresse, traîne de ville en ville, fréquente sa communauté, nourrit le rêve de revoir Cuba, de marcher dans cette Havane ensoleillée, éclatante, pleine de vie, trépidante telle une ruche (idem, 45). Faut-il qu'ils retournent dans leurs pays respectifs pour connaître enfin l'apaisement souhaité?

Les liesses qui ont suivi le départ du dictateur haïtien en exil, Normand les a vues de loin, seulement à la télévision. II connaîtra le trépas, une heure 22 minutes de vol d'Haïti, soit à une distance de 1097 kilomètres sans avoir posé les pieds sur le sol haïtien. Quant à Amparo, elle a fait le voyage pour s'apercevoir que son séjour cubain est vain et n'a été source que de déchirures et de tourments. Tout a changé dans son pays, les maisons, les rues, les rideaux, même les odeurs ne lui sont plus familières: "La magie avait quitté sa ville" (ibidem). Plus jamais elle ne pourra y retourner pour vivre, se dit-elle. D'où l'incontournable nécessité de vivre là où elle est.

Il en découle que le mal-être que connaissent les personnages n'est pas seulement dû à la nostalgie du sol natal, décrit avec un réalisme qui côtoie le merveilleux (Hoffmann, 1995). Quels réaménagements identitaires faudrait-il pour que le migrant s'adapte à sa nouvelle situation sans trop de déchirements intérieurs majeurs?

Ollivier, dans Passages (1992), rejette le principe d'une appartenance unique. Pour lui, seuls les simples d'esprit croient être originaires d'un lieu spécifique! Alors se pose, ici, avec acuité la question de la "double appartenance" ou des "appartenances multiples" (Spear, 2002). C'est-à-dire le fait qu'un individu partage deux appartenances, deux souverainetés, deux situations sociales et culturelles. Dans Passages, les personnages sont haïtiens, américains, italiens, cubains, juifs, métis. Mais leur destin se croise au Québec et ils coexistent dans province, et malgré leurs différences raciales et géographiques. Leyda, l'épouse de Normand garde en mémoire ce brassage épidermique dont elle a été témoin, lors des festivités carnavalesques, dans le quartier de Notre-Dame-de-Grâce à Montréal (Ollivier, 1992: 38).

Les personnages, avons-nous dit, sont doubles déjà du point de vue civil: Normand est Haïtien-Québécois. II voyage avec son passeport canadien, mais s'étonne de s'entendre dire à l'agent d'immigration qu'il est haïtien. Cette intégration (civique) dans son nouveau pays lui parait "une coquetterie de l'histoire (...)" (idem, 68). Car il reste Haïtien par sa peau, par son humour. Amparo cette autre figure emblématique de Passages est cubaine, de nationalité, car elle est fille de Syriens immigrés à la Havane. Elle vit au Canada depuis dix 
ans et habite Vancouver. Elle entretient une relation avec un Chilien et effectue de nombreux voyages en Amérique centrale, notamment au Nicaragua (idem, 40).

C'est entre déracinement et enracinement (ici et là-bas, dépaysement), entre déplacements et stabilité (aujourd'hui, hier et demain, etc.), entre soi et l'autre (moi: exilé, dépositaire, mémoire, oubli, l'hybride, etc.), entre ruptures et continuités (intérieures, extérieures, anciennes et nouvelles valeurs, cultures, identités, langues), entre déconstruction et construction que le migrant s'adapte à sa nouvelle situation. Ces interactions rejoignent la dynamique postmoderne.

\subsection{Fusionnement de la langue}

Comme nous l'avons mentionné précédemment, l'écrivain migrant parle aux moins deux langues: sa langue maternelle et sa langue d'adoption. Aussi, il joue sur deux plans linguistiques. Dans Passages, Émile Ollivier s'exprime et fait parler ses personnages dans un français qui est autant sien qu'elle est autant la langue de l'autre. Cette langue qui est pour lui comme elle l'est pour les écrivains beurs dont fait référence Collès (2007), une "langue d'échange et de circulation d'une diversité d'univers de référence" (ibidem) est aussi, qu'on le veuille ou non, dans une certaine mesure, "l'expression d'une identité culture". En effet, comme nous l'avons souligné précédemment, cette autre langue qu'est le français fait partie intégrante de l'identité d'Ollivier en tant qu'Haïtien (Hurbon, 1979; Lofficial, 1979; Saint-Germain, 1988) et de sa culture.

Passages est écrit dans une langue riche où se dégage une musicalité. Les personnages font un usage d'un fusionnement de langues où se regroupent le créole, le français et l'anglais et même un peu l'espagnol. Comme dans ses autres romans, Émile Ollivier confirme qu'il peut travailler dans la langue de "l'autre" ou de "l'hôte" (2002: 94), en maintenant son intonation à lui. Ainsi, les lecteurs font face à une langue ouverte, métissée, colorée, donc postmoderne. Cette coloration est une "revendication du respect de l'altérité".

Animal social, l'être humain se définit par le mouvement, intérieur et extérieur. Celuici est appelé à entrer en relation avec ses semblables, eux-mêmes en constants mouvements et en transformation. Ces mutations se reflètent dans la littérature migrante publiée au Québec et ailleurs. Ces écrivains, Naïm Kattan, Dany Laferrière, Ying Chen, Marco Micone et Émile Ollivier pour ne citer que ceux-ci, publient au Québec des écrits qui dépassent largement les limites géographiques de leur pays de naissance. Les matériaux qui constituent leurs écrits sont hétérogènes: les lieux sont divers; les cultures y sont plurielles: elles sont d'ici et de là-bas. Plus que doubles, les personnages sont le lieu de multiples appartenances. Imaginaires, styles et langues sont métissés. La langue d'adoption qu'ils utilisent est "reterritorialisée", c'est-à- dire ajustée, transformée pour chacun à sa 
manière personnelle. Pour toutes ces raisons, la littérature migrante s'inscrit dans le postmodernisme: elle peut être perçue comme un carrefour, un lieu de convergences où se croisent des personnages et des cultures s'entrechoquent et se transforment sans cesse. Quoiqu'il en soit, des pistes de recherches ultérieures pourraient s'orienter vers d'autres écrivains de la francophonie, par exemple, les comparer avec des écrivains migrants qui publient au Québec. En outre, qu'en est-il des écrivains migrants anglophones? Finalement, devrait-on continuer à parler de littérature migrante? Dans ce monde désenchanté, les romans ne devraient-ils pas nous présenter cette convergence de cultures comme une chance à saisir et non comme des déchirures? 


\section{Bibliographie}

Abdallah-Pretceille, Martine (1999). L'Éducation interculturelle. Paris: Presses Universitaires de France, collection "Que sais-je?".

BARTHES, Roland (1993). Le bruissement de la langue. Paris: Seuil.

BeRA, M. et LAMY, Y. (2003). Sociologie de la culture. Paris: Colin.

BERROUET-ORIOL, Robert (1986). "L'effet d'exil”. In: Vice versa, no 17, pp. 20-21.

BILLAUD, Jean (1996). L'homme et la culture: racines et perspectives. Lyon: Chronique Sociale.

BOUCHER, Louis-Philippe et MOROSE, Joseph (1990). "Responsabilisation et appartenance: la dynamique d'un projet éducatif ". In: Revue des sciences de l'éducation, vol.16, no 3, pp. 415-431.

BRUNEAU, M; BERTHOMIERE, W et CHIVALLON, C. (2006). Les diasporas dans le monde contemporain: un état des lieux. Paris: Kartala.

CAMILLERI, Carmel. (2004). "Cultures et stratégies ou les mille manières de s'adapter". In: Catherine. Halpern et Jean-Claude Ruano-Borbalan (dir.), Identité(s). L'individu, le groupe, la société. Auxerre: Éditions Sciences de l'homme.

CAMPEAU, Robert et al. (2004). Individu et société: Initiation à la sociologie. Montréal: Gaétan Morin Éditeur.

CECCON. Jérôme. (2004). "Les écritures migrantes au Québec". In: Africultures, no 59, Diaspos. www.africultures.com/index.asp?menu=revueaffichearticle\&no=3508 [consulté le 23/04/2006]

CHARLES J-C. (2001). “L’enracinnerrrance”. In: Boutures. vol. 1, n4 , pp. 37-41. http://www.lehman.cuny.edu/ile.en.ile/boutures/0104/charles.html [consulté le 24/03/2010].

ColLÈS, L, (2009). "Plaidoyer pour l'insertion de la littérature migrante à l'école" http://74.125.93.132/search?q=cache:ZG9g2d01OjIJ:alainindependant.canalblog.com/archives/200 9/02/02/12266720.html+La+langue+dans+la+litt\%C3\%A9rature+migrante\&cd $=1 \& \mathrm{hl}=\mathrm{fr} \& \mathrm{ct}=\mathrm{clnk} \& \mathrm{gl}=$ ca [consulté le 24/03/2010]

Colles, L. et LeBRUn, M. (2007). La littérature migrante dans l'espace francophone. Louvain: Éditions Modulaires Européennes.

FONKOUA, R. (1993). "Roman et poésie d'Afrique francophone: de l'exil et des mots pour le dire". In: Revue de littérature comparée, no 1, pp. 25-41.

FORGET, D. (2003). "Les nouveaux paradigmes de l'identité La littérature migrante au Québec". In: P. Ouellet (dir.), Le Soi et l'Autre. L'énonciation de l'identité dans les contextes interculturels. Québec: Presses de l'Université Laval.

GALATY, J. G. (1991). "Culture". In: Dictionnaire de l'ethnologie et de l'Anthropologie (Pierre Bonte et Michel Izard dir.), Paris: Presses Universitaires de France.

Guerraoul, Z., et Troadec, B. (2000). Psychologie interculturelle. Paris: Armand Colin.

GUERTIN, D. (1987). Sentiment d'appartenance chez l'adolescente et chez l'adolescent en milieu scolaire, Québec: Mémoire UQAM, 167 pp.

HAREL, S. (2005). Les passages obligés de l'écriture migrante. Montréal: XYZ éditeur, coll. r.

KRISTEVA, J. (1988). Etrangers à nous-mêmes. Paris: Fayard.

LEBRUN, M. (2001). "Visions transfrontalières du champ de la littérature migrante". In: L'Association pour la recherche interculturelle, $n^{\circ} 36, \mathrm{pp} .49-57$.

LÉVI-STRAuSS, C et al. (1987). L'Identité. Paris: Presses Universitaires de France,Quadrige.

LIPOVETSKY, G. (1990). "Virage culturel, persistance du moi”. In: Débat, n60, pp. 266-267.

MOISAN, C. et HILDEBRAND, R. (2001). Ces étrangers du dedans: une histoire de l'écriture migrante au Québec. Québec: Nota Bene.

MOLES, A. (1967). Sociodynamique de la culture. Paris: Mouton. 
MUCCHIELLI, R (1980). Le travail en groupe. Paris: Éditions ESF.

NEPVEU, P. (1999). L'écologie du réel: mort et naissance de la littérature québécoise contemporaine. Montréal: Boréal.

OLLIVIER, Emile (1984). "Quatre thèses sur la transculturation". In: Cahiers de recherches sociologiques, vol 2, pp. 75-90.

OLLIVIER, Emile (1999). "Et me voilà otage et protagoniste". In: Les Écrits, nº 95, pp. 4-7.

OLLIVIER, Emile (2002). "La langue sur l'établi”. In: Relations, décembre 2002, nº81.

OLLIVIER, Emile (2001). Repérages. Montréal: Leméac.

OUELLET, P. (2003). L'esprit migrateur: Essai sur le non-sens commun. Montréal: Trait-d'union.

Prud'Homme, N. (2002). La problématique identité collective et les littératures (im)migrantes au Québec (Mona Latif Ghattas, Antonio D’Alfonso et Marco Micone). Québec: Nota Bene.

RAULET, G. (1989). "Stratégies consensuelles et esthétique postmoderne". In: Recherche sociologique, vol. XX, $\mathrm{n}^{\circ}$ 2, p.11.

Rey-Debove, J., Cottez, H et Rey, L. (2005). Le nouveau Petit Robert, Dictionnaire alphabétique et analogique de la langue française. Paris: Firmin-Didot.

RoBIN, R. (1992). "Sortir de l'ethnicité". In: J-M. Lacroix et F. Caccia (dir.), Métamorphoses d'une utopie. Montréal: Triptyque, pp. 25-43.

SENGHOR, L. S. (1993). Liberté, 5, Le dialogue des cultures. Paris: Seuil. 
